Nervenarzt 2021 · 92:548-555

https://doi.org/10.1007/s00115-021-01093-1

Angenommen: 2. Februar 2021

Online publiziert: 29. März 2021

(c) Springer Medizin Verlag GmbH, ein Teil von Springer Nature 2021

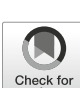

Check for
updates

Helmar C. Lehmann' • Benedikt Schoser ${ }^{2}$ Gilbert Wunderlich ${ }^{1,5} \cdot$ Peter Berlit ${ }^{3}$. Gereon R. Fink ${ }^{1,4}$

'Klinik und Poliklinik für Neurologie, Universitätsklinikum Köln, Köln, Deutschland

${ }^{2}$ LMU Klinikum, Friedrich-Baur-Institut, Neurologische Klinik und Poliklinik, Ludwig-Maximilians-

Universität München, München, Deutschland

${ }^{3}$ Deutsche Gesellschaft für Neurologie (DGN), Berlin, Deutschland

${ }^{4}$ Institut für Neurowissenschaften (INM-3), Forschungszentrum Jülich, Jülich, Deutschland

${ }^{5}$ Zentrum für Seltene Erkrankungen, Universitätsklinikum Köln, Köln, Deutschland

\title{
Neuromuskuläre Komplikationen einer SARS-CoV-2-Infektion - Teil 2: Erkrankungen der Muskulatur
}

Symptome bzw. Erkrankungen der Muskulatur und der neuromuskulären Transmission, die während oder nach einer COVID-19(„,coronavirus disease 2019“)Erkrankung beschrieben wurden, umfassen Myalgien, Myositiden, Rhabdomyolyse, Myasthenia gravis und „intensive care-unit(ICU)-acquired weakness" (ICUAW).

\section{Myalgien infolge COVID-19}

Myalgien sind ein häufiges und frühes Symptom während COVID-19 (ca. 35\% in der Studie von Wang und Kollegen [37] und über $50 \%$ bei Lechient et al. [25]). Dies ist vergleichbar mit der Häufigkeit von Myalgien bei der saisonalen Influenza $46 \%$ [32]. Bei etwa einem Drittel der Patienten ist eine erhöhte Kreatinkinase (CK) im Serum nachweisbar [21]. In etwa der Hälfte der Patienten bessern sich die Myalgien innerhalb weniger Tage, ähnlich wie die Symptome Fieber und Husten [26, 38].

Patienten, die Myalgien und eine erhöhte CK (> $3.3333 \mu \mathrm{mol} / \mathrm{sl})$ aufweisen, zeigen häufiger Leber- und Nierenschäden und haben einer chinesischen Studie zufolge ein höheres Risiko (Odds Ratio $[\mathrm{OR}]=2,56$, bei $\mathrm{CK}>3.0833 \mu \mathrm{mol} / \mathrm{sl}$ ) an COVID-19 zu versterben [27, 39]. Diese Assoziation wurde allerdings in einer vergleichbaren Studie aus New York nicht gefunden [10] und ist deutlich geringer im Vergleich zu anderen prognostisch relevanten Laborparametern, z. B. einer erhöhten Laktadehydrogenase $(\mathrm{OR}=37,52)$ oder einem erhöhten C-reaktiven Protein $(\mathrm{OR}=12,11$; [27]).

\section{Myositiden}

Aktuell sind wenige Fälle einer Myositis nach COVID-19 bekannt. Die Diagnose basierte überwiegend nur auf (unspezifischen) magnetresonanztomographischen (MRT-)Veränderungen [4, 29]. Eine kleine Fallserie aus Indien berichtet von 5 Patienten mit Dermatomyositis bei COVID-19-Patienten, die auf die gängigen Immuntherapien (Kortikoide, Immunglobuline) ansprachen [16].

\section{Rhabdomyolyse}

Rhabdomyolyse ist ein klinisches und biochemisches Syndrom, das durch eine akute Nekrose von Skelettmuskulatur ausgelöst wird. Klinisch bedeutsam ist eine Myoglobinurie, die in 15-33\% der Fälle zu einem Nierenversagen führt. In weniger als $5 \%$ der Fälle verläuft die Rhabdomyolyse tödlich. Virusinfektionen sind bei Kindern eine häufigere Ursache, bei Erwachsenen sind Substanzkonsum, Trauma und epileptische Anfälle häufige Auslöser, Virusinfektionen, bis auf Influenza A, hingegen sehr selten. In der Literatur sind mindestens 38 Fälle einer Rhabdomyolyse während oder nach einer COVID-19-Infektion beschrieben: Diese kann sowohl früh in der Erkrankungsphase, offenbar auch als erstes Symptom [35], als auch im späteren klinischen Verlauf $[5,11]$ auftreten. Die Serum-CK-Werte können dabei Werte bis $\mathrm{zu}$ mehr als $6688 \mu \mathrm{mol} / \mathrm{sl}$ im Verlauf annehmen [5]. Die Häufigkeit einer COVID-19-assoziierten Rhabdomyolyse ist nicht bekannt. Eine Arbeit aus China aus der Frühphase der Pandemie berichtet von 2 von 1099 Patienten [18]. Es scheint sich somit um eine seltenere Komplikation zu handeln.

\section{Merke}

Myalgien und Asthenie, die typischen Symptome einer Rhabdomyolyse, können durch Analgosedierung maskiert sein. Daher sollten regelmäßige CKKontrollen bei analogsedierten COVID19-Patienten erfolgen.

Bis November 2020 sind mindestens 38 Fälle einer COVID-19-assoziierten Rhabdomyolyse beschrieben worden, von denen 10 tödlich verliefen. Einschränkend muss hinzugefügt werden, dass bei einigen Patienten eine Komedikation mit möglicherweise myotoxischen 
Hier steht eine Anzeige.

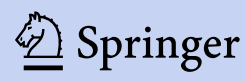




\section{Leitthema}

Tab. 1 Konzept der "intensive care unit acquired weakness". (Nach [3, 13])

Critical-illness-Polyneuropathie (CIP)

Critical-illness-Myopathie (CIM)

\begin{tabular}{|c|c|c|}
\hline Risikofaktoren & \multicolumn{2}{|c|}{$\begin{array}{l}\text { Sepsis, Multiorganversagen, weibliches Geschlecht, Multimorbidität, lange Dauer einer Organdysfunktion, langer Aufenthalt auf } \\
\text { Intensivstation, lange Beatmung auf der Intensivstation, Hyperglykämie (Kortikosteroide und Muskelrelaxanzien nicht konsistent } \\
\text { in allen Studien als Risikofaktor nachweisbar) }\end{array}$} \\
\hline Symptomatik & $\begin{array}{l}\text { Muskelschwäche } \\
\text { Verzögertes „Weaning" } \\
\text { Muskelatrophie }\end{array}$ & $\begin{array}{l}\text { Muskelschwäche } \\
\text { Verzögertes „Weaning“ } \\
\text { Muskelatrophie } \\
\text { CK erhöht }\end{array}$ \\
\hline Elektrophysiologie & Axonale sensomotorische Neuropathie & $\begin{array}{l}\text { SNAPa erhalten, } \\
\text { MSAP reduziert } \\
\text { EMG: myopathisch alterierte Potenziale }\end{array}$ \\
\hline $\begin{array}{l}\text { Biopsie (in der } \\
\text { Regel nicht not- } \\
\text { wendig) }\end{array}$ & Axonale Degeneration motorischer und sensibler Nervenfasern & Myopathie in Muskelbiopsie \\
\hline
\end{tabular}

Substanzen bestand, wie beispielsweise Imatinib [12, 33], Paclitaxel [11, 36] oder Rosuvastatin [1]. Histopathologische Untersuchungen bzw. elektromyographische (EMG-)Untersuchungen wurden in der Regel nicht durchgeführt. Es wird angenommen, dass viral vermittelte Rhabdomyolysen durch direkte virale Invasion der Muskeln hervorgerufen werden. In der Folge entstehen Muskelnekrosen durch virale Toxine oder durch eine aberrante Immunreaktion. SARS-CoV-2 infiziert den Wirt über den „Angiotensin-converting-enzyme-2"(ACE2)-Rezeptor. Der ACE2Rezeptor wird von Muskelzellen, aber auch Endothelzellen exprimiert, sodass eine direkte Infektion von Muskelzellen möglich ist $[2,19]$.

\section{"ICU acquired weakness"}

Unter dem Begriff der „ICU acquired weakness“ (ICUAW) werden eine Critical-illness-Polyneuropathie (CIP) und eine Critical-illness-Myopathie (CIM) zusammengefasst (• Tab. 1).

Obwohl beide Erkrankungen klinisch und elektrophysiologisch prinzipiell unterscheidbar sind, wird häufig von einer ICUAW oder Critical-illness-Polyneuropathie-Myopathie (CIPM) gesprochen, um der klinischen Überlappung (nicht zuletzt aufgrund erschwerter Diagnostik auf der Intensivstation) sowie dem Konzept eines gemeinsamen Pathomechanismus mit lediglich unterschiedlicher Organmanifestation Rechnung zu tragen (•Tab. 1).
Dieses beinhaltet eine gestörte $\mathrm{Mi}$ krozirkulation des Nerven- und Muskelgewebes sowie Dysfunktion von Mitochondrien, möglicherweise durch Hyperglykämien getriggert. Andere Faktoren, von denen angenommen wird, dass sie zur ICUAW beitragen, sind eine katabole Stoffwechsellage, systemische Inflammation und oxidativer Stress [24].

》) Es ist davon auszugehen, dass ICUAW eine häufige und relevante Komplikation bei COVID-19 ist

Die hohe Rate intensivpflichtiger Patienten, die eine oder mehrere Episoden einer Sepsis durchleiden, und die häufige Notwendigkeit einer prolongierten Beatmung legen nahe, dass bei COVID-19Patienten das Auftreten einer ICUAW eine klinisch besonders relevante Komplikation darstellen könnte. Bisher ist allerdings nicht systematisch untersucht, wie häufig ICUAW im Rahmen von COVID-19 auftritt. Neben Fallberichten gibt es eine Fallserie von 12 schwer erkrankten COVID-19-Patienten, die zwischen 12 und 49 Tagen auf einer Intensivstation behandelt wurden [6]. Diese berichtet über CIMkompatible klinische und elektrophysiologische Befunde bei 7 Patienten und bei 4 weiteren Kranken mit einer CIP. Bemerkenswert war der Nachweis ausgeprägter pathologischer Spontanaktivität im EMG bei vielen Patienten, während myopathische Potenziale weniger konsistent nachweisbar waren. Ähnliche EMG-Befunde wurden auch von einer Arbeitsgruppe aus Rom von 6 intubierten COVID-19-Patienten berichtet, wobei diese allerdings zusätzlich das Medikament Hydroxychloroquin erhalten hatten, welches eine toxische Myopathie induzieren kann $[8,28]$. Pathologische Spontanaktivität findet man häufig bei einer Myositis, allerdings sind die wenigen Berichte von Muskelbiospien bei COVID-19-Patienten nicht hinweisend auf floride Myositiden, sondern zeigen eher das Bild einer unspezifischen Muskelschädigung/Rhabdomyolyse mit Muskelfasernekrosen ohne Nachweis entzündlicher Infiltrate im Muskel bzw. in den Gefäßen [6].

Einer Muskelbeteiligung bei SARSCoV-2-Infektionen mit einem Spektrum von Myalgien über Rhabdomyolyse bis hin zu einer CIPM muss nicht zwingend eine gemeinsame Pathogenese zugrunde liegen. Vorstellbar wäre eine direkte Infektion von Muskelzellen, die - wenn auch in niedriger Frequenz - den ACE2Rezeptor exprimieren [2, 19], über den das SARS-CoV-2-Virus in die Zelle gelangt. Der ACE2-Rezeptor wird von Endothelzellen ebenfalls exprimiert, sodass auch eine Vaskulitis mit primärer Affektion des Muskels vorstellbar wäre [23].

\section{Merke}

COVID-19 geht mit bekannten Risikofaktoren einer Critical-illness Polyneu- 
ropathie bzw. Myopathie (CIPM) einher. Systematische Untersuchungen über die Häufigkeit von CIPM bei COVID-19 fehlen allerdings bisher.

\section{Myasthenia gravis}

Zwei Fallberichte existieren von einer 2 bzw. 4 Wochen nach einer milden COVID-19-Infektion neu aufgetretenen okulären Myasthenia gravis [22, 34]. Die Diagnose wurde aufgrund einer typischen Symptomatik und dem Nachweis erhöhter Acetylcholinrezeptorantikörper gestellt [22, 34]. Therapeutisch wurden erfolgreich Pyridostigmin und intravenöse Immunglobuline (IVIg) eingesetzt. Eine Fallserie aus Italien beschreibt zudem 3 Patienten, bei denen sich eine generalisierte Myasthenia gravis 5 bis 7 Tage nach Infektion mit SARSCoV-2 entwickelte. Bei allen Patienten wurden Acetylcholinrezeptorantikörper nachgewiesen. Die Behandlung erfolgte mit Prednisolon, IVIg oder Plasmapherese und führte zu einem Rückgang der Symptome bei allen Patienten [30].

\section{) Fieberhafte Infekte können zur klinischen Erstmanifestation einer Myasthenie führen}

Es ist durchaus denkbar, dass die SARSCoV-2-Infektion eine bislang subklinische Myasthenie „demaskiert", so wie auch andere fieberhafte Infekte zur klinischen Erstmanifestation einer Myasthenie führen können. Auf der anderen Seite weisen Scopelliti und Mitarbeiter auf das Risiko der Fehldiagnose einer COVID19-Infektion bei Patienten mit neu aufgetretener Myasthenie hin. Sie berichten über einen Patienten, bei dem trotz mehrfach negativer SARS-CoV-2-Polymerase-Kettenreaktion(PCR) eines $\mathrm{Na}$ sen-Rachen-Abstrichs eine COVID-19Infektion ausschließlich aufgrund einer respiratorischen Dysfunktion und dem Verdacht auf eine leichte interstitielle Pneumonie in einem Thoraxröntgenbild diagnostiziert wurde. Erst später traten Doppelbilder und eine Hypophonie auf, sodass letztlich die Diagnose einer (seronegativen) bulbären Manifestation einer Myasthenia gravis u.a. durch Nach-

Nervenarzt 2021 - 92:548-555 https://doi.org/10.1007/s00115-021-01093-1

(c) Springer Medizin Verlag GmbH, ein Teil von Springer Nature 2021

\section{H. C. Lehmann · B. Schoser · G. Wunderlich · P. Berlit · G. R. Fink}

\section{Neuromuskuläre Komplikationen einer SARS-CoV-2-Infektion - Teil 2: Erkrankungen der Muskulatur}

\section{Zusammenfassung}

Neben Störungen und Erkrankungen peripherer Nerven sind in den letzten Monaten im Zusammenhang mit COVID-19 ("coronavirus disease 2019") auch Begleitsymptome und Störungen der Muskulatur bzw. der neuromuskulären Transmission beschrieben worden. Im zweiten Teil unserer Zusammenfassung geben wir eine Übersicht über häufig berichtete Symptome wie Myalgien und definierte Erkrankungen wie Rhabdomyolysen, Myositiden, Myasthenie und "intensive care unit acquired weakness" (ICUAW), die im Rahmen eine SARS-CoV2(, severe acute respiratory syndrome coronavirus 2")-Infektion bzw. COVID-19 beschrieben wurden. Darüber hinaus werden Kriterien für eine Kausalität wie Effektstärke, Plausibilität, Zeitverlauf und experimentelle Evidenz für einen kausalen Zusammenhang der in beiden Teilen der Übersicht beschriebenen COVID-19 assoziierten neuromuskulären Erkrankungen diskutiert. Zum jetzigen Zeitpunkt sind - neben der auch in der Laienpresse bekannten Geruchssinnstörung - vor allem Myalgien als unspezifisches Symptom häufige Folge einer symptomatischen SARS-CoV-2 Infektion. Andere neuromuskuläre Komplikationen erscheinen hinsichtlich ihrer Pathogenese prinzipiell plausibel, aber offenbar selten Folge einer SARS-CoV-2-Infektion zu sein. Prospektive bzw. Kohortenstudien sind notwendig, um eine Kausalität zu bestätigen und das Risiko abzuschätzen.

\section{Schlüsselwörter}

Muskelkrankheiten [C05.651] · Neuromuskuläre Manifestationen [C10.597.613] . Ätiologie · Kausalität [N05.715.350.200] . Molekulare Mimikry [G02.111.560]

\section{Neuromuscular complications of SARS-CoV-2 infection-Part 2: muscle disorders}

\section{Abstract}

Apart from disorders and diseases of the peripheral nerves, symptoms and disorders of the musculature and the neuromuscular transmission have also been described in association with coronavirus disease 2019 (COVID-19). In the second part of our review we provide an overview about frequently reported symptoms, such as myalgia as well as defined disorders, such as rhabdomyolysis, myositis, myasthenia and intensive care unit (ICU)-acquired weakness, which have been described during severe acute respiratory syndrome coronavirus 2 (SARS-CoV-2) infections or COVID-19.Furthermore, the criteria for a causality, such as association strength, plausibility, time course, and experimental evidence for a causal association that should be applied for the COVID-19-asssociated neuromuscular conditions described in the two parts of the review are discussed. At present, in addition to anosmia, which is also known in the lay press, myalgia in particular as a nonspecific symptom are frequent sequelae of a symptomatic SARS-CoV-2 infection. Other neuromuscular complications seem to be principally plausible (considering the pathogenesis) but apparently rare consequences of a SARS-CoV-2 infection. Prospective or cohort studies are necessary to confirm a causality and assess the risk.

Keywords

Muscular diseases [C05.651] - Neuromuscular manifestations [C10.597.613] · Etiology · Causality [N05.715.350.200] · Molecular mimicry [G02.111.560] weis eines Dekrements gestellt wurde [31]. Bei Patienten mit bekannter Myasthenie, die an COVID-19 erkranken, solle individuell abgewogen werden, die entsprechende Therapie beizubehalten oder zu modifizieren. So wird z. B. ein Wiederanstieg der Lymphozytenzahlen nach Absetzen von Azathioprin erst 3 bis
5 Wochen später zu erwarten sein [7]. Im Gegensatz dazu besteht das Risiko, eine durch COVID-19-bedingte myasthene Krise hervorzurufen. 


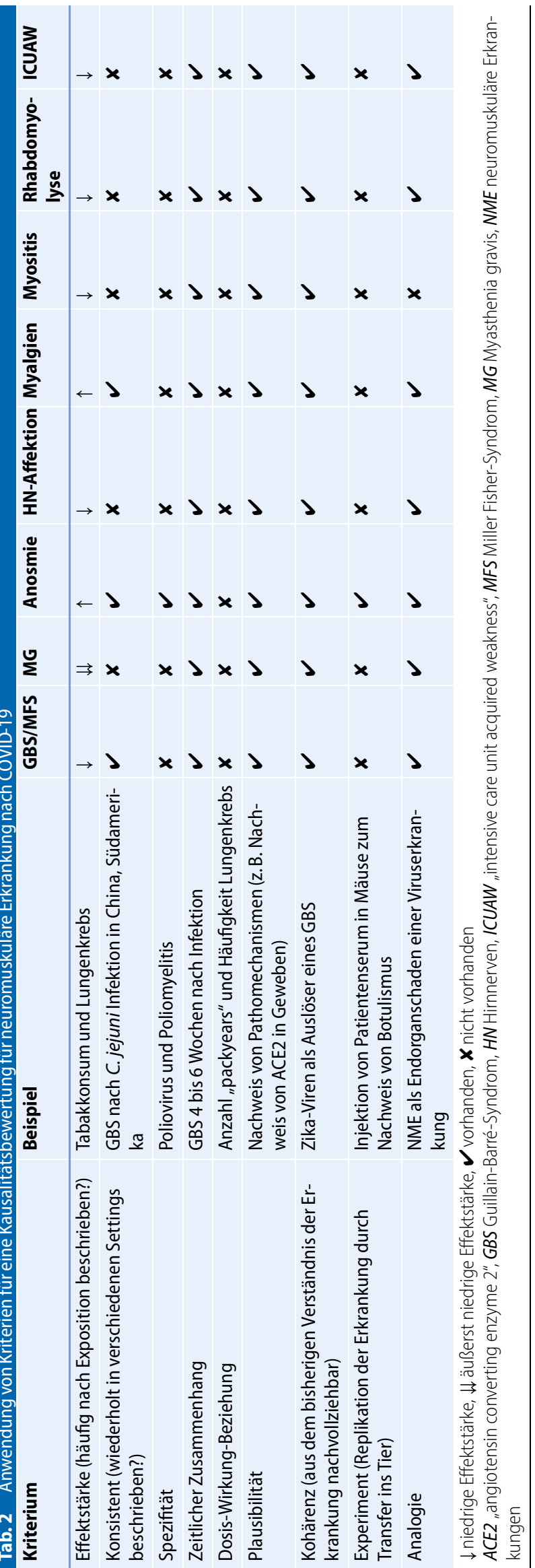

Cave

Aus den bisherigen Fallberichten zur Myasthenia gravis bei SARS-COV-2Infektion lässt sich ableiten, dass:

- die Erstdiagnose einer Myasthenie nach COVID-19 sehr selten ist,

- sowohl okuläre als auch generalisierte Myasthenien auftreten können,

- in der Regel Acetylcholinrezeptorantikörper nachweisbar waren,

- die Myasthenie auf die gängigen Therapien anspricht.

\section{Neuro-COVID oder Publikati- onsbias? Objektive Bewertung notwendig}

Die Vielzahl von Publikationen und klinischen Daten über typische und seltenere außerpulmonale Manifestationsformen in den letzten Monaten dokumentiert den rasanten Zuwachs an Wissen bezüglich SARS-CoV-2 und COVID-19. So erfreulich dies ist, so ist auf der anderen Seite nicht auszuschließen, dass nicht zuletzt durch "calls for COVID-19 papers" von Herausgebern und „Open-access“Initiativen für COVID-19-Artikel von Wissenschaftsverlagen das tatsächliche Ausmaß des Spektrums neurologischer Begleiterscheinungen nach COVID19 falsch eingeschätzt werden könnte, zumal das obligate „Peer-Review“-Verfahren vor allem zu Beginn der Pandemie nicht konsequent eingesetzt wurde. Um eine Kausalität abzuleiten, plädieren wir dafür, dass Evidenzkriterien formuliert werden, wie sie beispielsweise bei Impfkomplikationen oder bei nichtinfektiösen Erkrankungen definiert wurden [9, 14,20]. Grundlage sind epidemiologische Faktoren und mechanistische bzw. experimentelle Nachweise ( $\bullet$ Tab. 2). Keines dieser Kriterien ist für sich genommen beweisend oder muss zwingend erfüllt sein; die Bewertung erfolgt in der $\mathrm{Zu}$ sammenschau. Fallberichte, als „kleinste publizierbare Einheit in der Medizinliteratur" [17] sind wichtig, um Hypothesen $\mathrm{zu}$ generieren, sollten aber dann auch ausreichende klinische Informationen (demographische Daten, Vordiagnosen, konkurrierende Expositionen, Einzelheiten der Erkrankung, klarer zeitlicher 
Hier steht eine Anzeige.

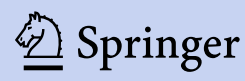


Zusammenhang, Häufigkeit und Dauer der Exposition) beinhalten [9], was natürlich auch für Fallserien gilt. So findet sich z. B. eine Fallserie von Patienten mit Guillain-Barré-Syndrom, bei denen über COVID-19 als Trigger spekuliert wurde, 7 von 8 Patienten aber weder einen SARS-COV-2-positiven Abstrich, noch Antikörper, noch einen kompatiblen Befund in der Thoraxcomputertomographie hatten [15]!

Wenn man obige Kriterien auf die im ersten (s. Neuromuskuläre Komplikationen einer SARS-CoV-2-Infektion Teil 1: periphere Nerven in dieser Ausgabe von Der Nervenarzt) und zweiten Teil (Teil 2: Erkrankungen der Muskulatur) dieser Übersicht beschriebenen neuromuskulären Komplikationen nach COVID-19 anlegt, so wird klar, dass diese zwar grundsätzlich plausibel und auch kohärent, d. h. mit dem Krankheitskonzept einer para- bzw. postinfektiösen Genese vereinbar sind. Allerdings zeigt sich ein sehr heterogenes Bild hinsichtlich Häufigkeit und Konsistenz: Bei über 100 Mio. bekannten SARS-CoV-2-Infektionen sind lediglich Anosmie und Myalgien als häufige Folge beschrieben. Diese sind (neben einem GBS) auch konsistent in unterschiedlichen Regionen von verschiedenen Autorengruppen beschrieben worden. Alle anderen Erkrankungen waren hingegen bisher selten. Im Gegensatz dazu scheint ICUAW infolge von COVID-19 sehr plausibel, allerdings gibt es (bisher) hierüber nur wenige valide Daten, weswegen ein COVID19-assoziiertes ICUAW möglicherweise unterdiagnostiziert sein könnte.

Daher sind prospektive Studien unabdingbar, um das Risiko für das Auftreten (auch seltenerer) neuromuskulären $\mathrm{Ne}$ benwirkungen und gegebenenfalls den daraus resultierenden vermehrten Therapie- und Rehabilitationsbedarf besser einschätzen zu können.

\section{Fazit für die Praxis}

- Die Muskulatur scheint bei COVID-

19 („,coronavirus disease 2019“) am häufigsten in Form von Myalgien symptomatisch betroffen zu sein. In seltenen Fällen scheinen offenbar auch Rhabdomyolysen auftreten zu können, die mit höherer Mortalität vergesellschaftet waren. Myasthenie und andere Erkrankungen der Muskulatur, wie z. B. Myositiden, sind bisher nur ganz vereinzelt beschrieben worden.

- Zur abschließenden Beurteilung, ob neuromuskuläre Komplikationen kausal durch COVID-19 bedingt sind, müssen erst die Ergebnisse prospektiver Studien abgewartet werden. Dafür sind bereits verschiedene Register in Deutschland und Europa entstanden, z. B. das Nationale Pandemie Kohorten Netz (NAPKON) und das Lean European Open Survey for SARS-CoV-2 Infected Patients (LEOSS-)Register, die durch einen zu erwartenden Einschluss einer großen Anzahl von Patienten auch geeignet wären, seltenere neurologische Folgeerscheinungen von COVID-19 zu erfassen.

\section{Korrespondenzadresse}

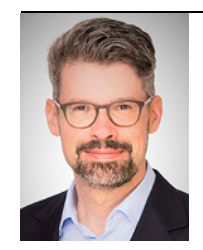

Prof. Dr. Helmar C. Lehmann Klinik und Poliklinik für Neurologie, Universitätsklinikum Köln Kerpener Straße 62, 50937 Köln, Deutschland helmar.lehmann@ uk-koeln.de

\section{Einhaltung ethischer Richtlinien}

Interessenkonflikt. H.C. Lehmann, B. Schoser, G. Wunderlich, P. Berlit und G.R. Fink geben an, dass kein Interessenkonflikt besteht.

Für diesen Beitrag wurden von den Autoren keine Studien an Menschen oder Tieren durchgeführt. Für die aufgeführten Studien gelten die jeweils dort angegebenen ethischen Richtlinien.

\section{Literatur}

1. Anklesaria Z, Frankman J, Gordin J et al (2020) Fatal rhabdomyolysis in a COVID-19 patient on rosuvastatin. Cureus 12:e11186. https://doi.org/ 10.7759/cureus.11186

2. Azizi SA, Azizi S-A (2020) Neurological injuries in COVID-19 patients: direct viral invasion or a bystander injury after infection of epithelial/ endothelial cells. J Neurovirol 26:631-641. https:// doi.org/10.1007/s13365-020-00903-7
3. Bednarík J, Vondracek P, Dusek L et al (2005) Risk factors for critical illness polyneuromyopathy. J Neurol 252:343-351. https://doi.org/10.1007/ s00415-005-0654-X

4. Beydon M, Chevalier K, Al Tabaa O et al (2020) Myositis as a manifestation of SARS-CoV-2. Ann Rheum Dis. https://doi.org/10.1136/annrheumdis-2020217573

5. Buckholz AP, Kaplan A, RosenblattRE, Wan D (2020) Clinical characteristics, diagnosis, and outcomes of 6 patients with COVID-19 infection and Rhabdomyolysis. Mayo Clin Proc 95:2557-2559. https://doi.org/10.1016/j.mayocp.2020.09.005

6. Cabañes-Martínez L, Villadóniga M, GonzálezRodríguez L, Araque L, Díaz-Cid A, Ruz-Caracuel I, Pian H, Sánchez-Alonso S, Fanjul S, del Álamo M, Regidor I (2020) Neuromuscular involvement in COVID-19 critically ill patients. Clin Neurophysiol 131(12):2809-2816

7. Campbell AC, Skinner JM, Hersey P et al (1974) Immunosuppression in the treatment of inflammatory bowel disease. I. Changes in lymphoid subpopulations in the blood and rectal mucosa following cessation of treatment with azathioprine. Clin Explmmunol 16:521-533

8. de Carvalho AAS (2020) Side effects of chloroquine and hydroxychloroquine on skeletal muscle: a narrative review. Curr Pharmacol Rep. https://doi. org/10.1007/s40495-020-00243-4

9. Committee to Review Adverse Effects of Vaccines, Institute of Medicine (2011) Adverse effects of vaccines: evidence and causality. National Academies Press, Washington (DC)

10. Cummings MJ, Baldwin MR, Abrams D et al (2020) Epidemiology, clinical course, and outcomes of critically ill adults with COVID-19 in New York City: a prospective cohortstudy. Lancet 395:1763-1770. https://doi.org/10.1016/S0140-6736(20)31189-2

11. Cunha M, Pinho I, Lopes M et al (2020) A case of corticosteroid-responsive SARS-CoV-2 related massive rhabdomyolysis. IDCases 22:e946. https:// doi.org/10.1016/j.idcr.2020.e00946

12. Damaraju VL, Kuzma M, Cass CE et al (2018) Multitargeted kinase inhibitors imatinib, sorafenib and sunitinib perturb energy metabolism and cause cytotoxicity to cultured $\mathrm{C} 2 \mathrm{C} 12$ skeletal muscle derived myotubes. Biochem Pharmacol 155:162-171. https://doi.org/10.1016/j.bcp.2018. 07.001

13. De Jonghe B, Sharshar T, Lefaucheur J-P et al (2002) Paresis acquired in the intensive care unit: a prospective multicenter study. JAMA 288:2859-2867. https://doi.org/10.1001/jama. 288.22.2859

14. Ellul M, Varatharaj A, Nicholson TR et al (2020) Defining causality in COVID-19 and neurological disorders. J Neurol Neurosurg Psychiatry 91:811-812. https://doi.org/10.1136/jnnp-2020323667

15. Gigli GL, Bax F, Marini A et al (2020) Guillain-Barré syndrome in the COVID-19 era: just an occasional cluster? J Neurol. https://doi.org/10.1007/s00415020-09911-3

16. Gokhale Y, Patankar A, Holla U et al (2020) Dermatomyositis during COVID-19 pandemic (A case series): is there a cause effect relationship? JAssoc Physicians India 68:20-24

17. Grimes DA, Schulz KF (2002) Descriptive studies: what they can and cannot do. Lancet 359:145-149. https://doi.org/10.1016/S0140-6736(02)07373-7

18. Guan W-J, Ni Z-Y, Hu Y et al (2020) Clinical characteristics of coronavirus disease 2019 in China. N Engl J Med 382:1708-1720. https://doi. org/10.1056/NEJMoa2002032 
19. Han T, Kang J, Li G, Ge J, Gu J (2020) Analysis of 2019-nCoV receptor ACE2 expression in different tissues and its significance study. Ann Transl Med 8(17):1077-1077

20. Hill $A B$ (1965) The environment and disease: association or causation? Proc R Soc Med 58:295-300

21. Huang C, Wang Y, Li X et al (2020) Clinical features of patients infected with 2019 novel coronavirus in Wuhan, China. Lancet 395:497-506. https://doi. org/10.1016/S0140-6736(20)30183-5

22. Huber M, Rogozinski S, Puppe $W$ et al (2020) Postinfectious onset of myasthenia Gravis in a COVID-19 patient. Front Neurol 11:576153. https://doi.org/10.3389/fneur.2020.576153

23. IbaT,Connors JM, LevyJH(2020) The coagulopathy, endotheliopathy, and vasculitis of COVID-19. Inflamm Res 69:1181-1189. https://doi.org/10. 1007/s00011-020-01401-6

24. Kress JP, Hall JB (2014) ICU-acquired weakness and recovery from critical illness. $\mathrm{N}$ Engl J Med 370:1626-1635. https://doi.org/10.1056/ NEJMra1209390

25. Lechien JR, Chiesa-Estomba CM, De Siati DR et al (2020) Olfactory and gustatory dysfunctions as a clinical presentation of mild-to-moderate forms of the coronavirus disease (COVID-19): a multicenter European study. Eur Arch Otorhinolaryngol 277:2251-2261. https://doi.org/10.1007/s00405020-05965-1

26. Liguori C, Pierantozzi M, Spanetta M et al (2020) Subjective neurological symptoms frequently occur in patients with SARS-CoV2 infection. Brain Behav Immun 88:11-16. https://doi.org/10.1016/ j.bbi.2020.05.037

27. Lu L, Zhong W, Bian Z et al (2020) A comparison of mortality-related risk factors of COVID-19, SARS, and MERS: a systematic review and meta-analysis. JInfect 81:e18-e25. https://doi.org/10.1016/j.jinf 2020.07.002

28. Madia F, Merico B, Primiano G et al (2020) Acute myopathic quadriplegia in patients with COVID-19 in the intensive care unit. Neurology 95:492-494. https://doi.org/10.1212/WNL. 0000000000010280

29. Mehan WA, Yoon BC, Lang Metal (2020) Paraspinal myositis in patients with COVID-19 infection. Ajnr Am J Neuroradiol 41:1949-1952. https://doi.org/ 10.3174/ajnr.A6711

30. Restivo DA, Centonze D, Alesina A, MarcheseRagona R (2020) Myasthenia Gravis associated with SARS-coV-2 infection. Ann Intern Med. https://doi.org/10.7326/L20-0845

31. Scopelliti G, Osio M, Arquati M, Pantoni L (2020) Respiratory dysfunction as first presentation of myasthenia gravis misdiagnosed as COVID-19. Neurol Sci. https://doi.org/10.1007/s10072-02004826-3

32. Shiley KT, Nadolski G, Mickus T et al (2010) Differences in the epidemiological characteristics and clinical outcomes of pandemic (H1N1) 2009 influenza, compared with seasonal influenza. InfectControlHospEpidemiol31:676-682.https:// doi.org/10.1086/653204

33. Solís JG, Esquivel Pineda A, Alberti Minutti P, Albarrán Sánchez A (2020) Case report: rhabdomyolysis in a patient with COVID-19: a proposed diagnostic-therapeutic algorithm. Am J Trop Med Hyg 103:1158-1161. https://doi.org/10.4269/ ajtmh.20-0692

34. Sriwastava S, Tandon M, Kataria S et al (2020) New onset of ocular myasthenia gravis in a patient with COVID-19: a novel case report and literature review. J Neurol. https://doi.org/10.1007/s00415020-10263-1

35. Tram N, Chiodini B, Montesinos I et al (2020) Rhabdomyolysis and Acute Kidney Injury as Leading COVID-19 Presentation in an Adolescent. Pediatr Infect Dis J 39:e314-e315. https://doi.org/ 10.1097/INF.0000000000002853

36. Vicente E, Zafra M, Garcia-Martinez E, de la Peña FA (2009) Acute rhabdomyolysis as a complication of paclitaxel-gemcitabine chemotherapy for ovarian cancer. Eur J Obstet Gynecol Reprod Biol 145:226 https://doi.org/10.1016/j.ejogrb.2009.04.029

37. Wang D, Hu B, Hu C et al (2020) Clinical characteristics of 138 hospitalized patients with 2019 novel Coronavirus-infected pneumonia in Wuhan, China. JAMA 323:1061-1069. https://doi. org/10.1001/jama.2020.1585

38. Zhang J, Wang X, Jia X et al (2020) Risk factors for disease severity, unimprovement, and mortality in COVID-19 patients in Wuhan, China. Clin Microbio Infect 26:767-772. https://doi.org/10.1016/j.cmi. 2020.04.012

39. Zhou F, Yu T, Du R et al (2020) Clinical course and risk factors for mortality of adult inpatients with COVID-19 in Wuhan, China: a retrospective cohort study. Lancet 395:1054-1062. https://doi.org/10. 1016/S0140-6736(20)30566-3
Springer Medizin Podcast

Medizin für Gesundheitsprofis

Der Podcast von SpringerMedizin.de geht seit Juli 2020 spannenden Fragen aus der Welt der Medizin nach immer freitags erweitert eine neue Folge das bereits bestehende OnlineAngebot.

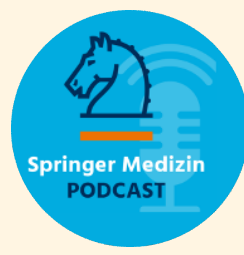

"Mit unserem

Podcast möch-

ten wir all jene ansprechen, die sich auf medizinische Themen in einer, gewissen

Flughöhe' einlassen möchten", erklärt Dr. Erik Heintz, Chefredakteur von SpringerMedizin.de das neue Format. "Gemeint sind damit Menschen, die sich nicht mit medizinischem Halbwissen zufrieden geben und gerne mehr wissen möchten. Daher legen unsere Redakteurinnen und Redakteure in München und Heidelberg vor allem auf den inhaltlichen Anspruch großen Wert. Unter Bezugnahme auf neue Studien, neue Erkenntnisse und praxisrelevantes Wissen bereiten wir jeden Podcast gründlich vor. Die Themen drehen sich um Gesundheit, Krankheit, Diagnostik, Therapie und Prävention, zu denen wir unsere Fachexpertinnen und Fachexperten aus verschiedenen medizinischen Fachgebieten hinzuziehen."

So entstehen abwechslungsreiche Folgen, die sowohl detailliert Leitlinien abarbeiten, der Evidenz von neuen Studienergebnissen auf den Grund gehen, aber auch die Hintergründe zu Erkrankungen und Therapien aufschlüsseln - sodass einerseits die ärztliche Praxis erleichtert und konkrete Tipps für die alltägliche Arbeit vermittelt werden, andererseits aber auch der Horizont über das eigene Fachgebiet hinaus erweitert werden kann.

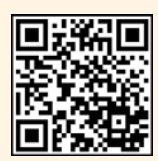

www.springermedizin.de/podcast 